\title{
Revascularização da artéria coronária direita intra-atrial
}

\author{
Artur LOURENÇĀO JÚNIOR*, Luís Alberto DALLAN*, Sérgio Almeida de OLIVEIRA*, Adib D. JATENE*
}

RBCCV 44205-122

\begin{abstract}
LOURENÇĀO JÚNIOR, A.; DALLAN, L. A.; OLIVEIRA, S. A.; JATENE, A. D. - Revascularização da artéria coronária direita intra-atrial. Rev. Bras. Cir. Cardiovasc. 5(3): 179-182, 1990.

RESUMO: A artéria coronária direita, em seu trajeto no sulco atrioventricular direito, pode, em raras ocasiōes, penetrar na cavidade atrial direita. Esta variaçāo anatômica poderá modificar a tática cirúrgica em operaçōes de revascularização miocárdica. No presente trabalho, relatamos o caso em que a ponte de veia safena para a artéria coronária direita foi realizada em posiçāo intra-atrial direita.
\end{abstract}

DESCRITORES: artéria coronária, revascularização; miocárdio, revascularização, cirurgia.

\section{INTRODUÇĀO}

Estudos anatômicos ${ }^{6}$ mostraram que em aproximadamente $0,1 \%$ da população a artéria coronária direita pode ter um percurso intracavitário. Nestas condiçōes, a artéria coronária direita penetra no átrio direito, em uma extensāo ao redor de 2 a 3 $\mathrm{cm}$, retornando à sua posiçāo subepicárdica no sulco atrioventricular.

Esta variaçāo anatômica pode acarretar dificuldades para o cirurgiāo, em operaçōes de revascularizaçāo miocárdica.

Neste trabalho, relatamos o caso de um paciente submetido a revascularizaçāo do miocárdio que apresentava essa anomalia coronária, sendo a ponte de safena para a artéria coronária direita realizada dentro da cavidade atrial direita.

\section{RELATO DO CASO}

Paciente de 58 anos, sexo masculino, advogado, diabético, com quadro de angina aos esforços de caráter progressivo há aproximadamente 2 meses e apresentando teste ergométrico positivo para isquemia miocárdia. Submeteu-se a cinecona- riografia (Figura 1) em 25/04/85, que mostrou lesāo obstrutiva de $70 \%$ em coronária direita no $1 / 3$ inical e médio, lesão segmentar de $95 \%$ em artéria descendente anterior ( $1 / 3$ inicial), comprometendo o primeiro e o segundo ramos diagonais, e lesão de $90 \%$ em artéria circunflexa e de $60 \%$ na origem do ramo marginal esquerdo.

Em 30/04/85 foi operado, sendo realizada revascularizaçāo do miocárdio, com anastomose término-lateral da artéria torácica interna esquerda com a artéria descendente anterior e pontes de veia safena para o primeiro e o segundo ramos diagonais, artéria marginal esquerda e artéria coronária direita, sendo a ponte para os ramos diagonais feita com anastomoses seqüenciais.

A operaçāo foi efetuada através de toracotomia mediana longitudinal, circulação extracorpórea com perfusāo arterial pela aorta ascendente e drenagem venosa através de canulaçāo de ambas as veias cavas, hipotermia leve $\left(32^{\circ} \mathrm{C}\right)$ e pinçamento intermitente da aorta.

As pontes de veia safena para as artérias diagonais e marginal esquerda, assim como a anastomose da artéria torácica interna esquerda com a artéria descendente anterior, foram realiza-

Trabalho realizado no Instituto do Coração do Hospital das Clínicas da Faculdade de Medicina da Universidade de São Paulo. São Paulo, SP. Brasil. Recebido para publicação em 5 de outubro de 1990.

- Do Instituto do Coraçáo do Hospital das Clínicas da Faculdade de Medicina da Universidade de Săo Paulo.

Endereço para separatas: Artur Lourençáo Júnior. Av. Dr. Enéas Carvalho de Aguiar, 44. Divisão Cirúrgica. 05403 São Paulo, SP. Brasil. 
LOURENÇĀo JÚNIOR, A.; DALLAN, L. A.; OLIVEIRA, S. A.; JATENE, A. D. - Revascularizaçāo da artéria coronária direita intra-atrial. Rev. Bras. Cir. Cardiovasc. 5(3): 179-182, 1990.
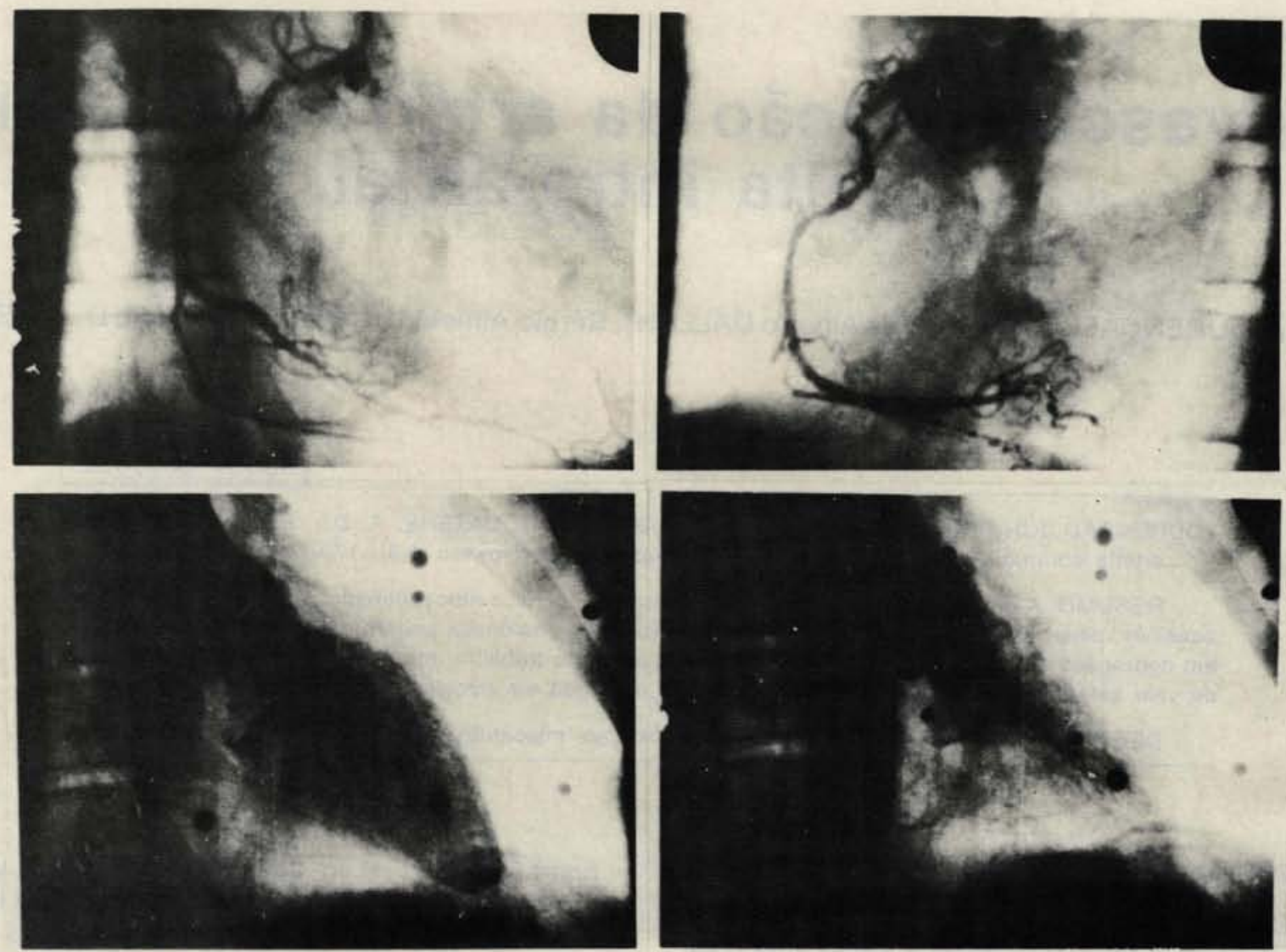

Fig. 1 - F.A.S. - 58a. - Masc. - Mulato - RG. 5.039.896-H - Cinecoronariografia pré-operatória, mostrando a artéria coronária direita em $O A D$ e $O A E$ e ventriculografia esquerda em sístole e diástole.

das de modo habitual, sem qualquer particularidade. Entretanto, a artéria coronária direita, na metade distal do tronco principal, foi exaustivamente procurada no sulco atrioventricular direito, não sendo identificada. Feita pequena incisão no átrio direito, junto ao sulco atrioventricular, após a margem aguda do coraçāo, foi identificada a artéria coronária direita cursando trajeto intracavitário. A anastomose da veia safena com a artéria coronária direita foi aí realizada (Figura 2). Em seguida, a atriotomia direita foi suturada, exteriorizando a veia safena pela extremidade superior da incisāo. A adventícia da veia safena foi frouxamente suturada nas bordas da atriotomia direita (Figura 3 ). A extremidade proximal do enxerto de veia safena foi, então, anastomosada na parede anterior da aorta.

A evoluçăo pós-operatória transcorreu sem intercorrências e, antes da alta hospitalar, foi realizada nova cinecoronariografia para controle ( $\mathrm{Fi}$ gura 4), que mostrou estarem pérvias as anastomoses realizadas e haver discreta consíricçāo da veia safena anastomasada à artéria coronária direita, no local onde foi exteriorizada no átrio direito. O paciente recebeu alta hospitalar no $10^{\circ}$ dia de pós-operatório, estando atualmente as- sintomático, do ponto de vista cardiovascular.

\section{COMENTÁRIOS}

$\mathrm{Na}$ literatura, há poucas publicaçōes relatando



Fig. 2 - F.A.S. - 58a. - Masc. - Mulato - RG. 5.039.896-H - Ponte de veia safena para artéria coronária direita intra-atrial (detalhe do término da anastomose, mostrando atriotomia direita com bordas afastadas por 2 pinças). 
LOURENÇĀO JÚNIOR, A.; DALLAN, L. A.; OLIVEIRA, S. A.; JATENE, A. D. - Revascularizaçāo da artéria coronária direita intra-atrial. Rev. Bras. Cir. Cardiovasc. 5(3): 179-182, 1990.



Fig. 3 - F.A.S. - 58a. - Masc. - Mulato - RG. 5.039.896-H - Ponte de veia safena para artéria coronária direita (aspecto após sutura da atriotomia).

o percurso intracavitário das artérias coronárias 4.5 . 9. 10 , sendo, por outro lado, grande o volume de trabalhos sobre artéria coronária com trajeto intramiocárdico 2, 3, 6-8, 11 .

A primeira citação da localizaçāo intracavitária das artérias coronárias é atribuída a MCALPINE ${ }^{5}$, onde, em dissecção de 1000 coraçōes normais, encontrou um caso no qual a coronária direita apresentava posicionamento intra-atrial direito $(0,1 \%)$ e três casos em que a artéria coronária descendente anterior posicionava-se dentro do ventrículo direito $(0,3 \%)$

OKSNER \& MILLS ${ }^{9}$ apresentaram, em 1984, relato de 13 pacientes com artéria coronária intracavitária, em um total de 4414 operaçōes de revascularização do miocárdio, dando uma incidência de $0,09 \%$ dentro de átrio direito (artéria coronária direita) e $0,2 \%$ dentro do ventrículo direito (artéria descendente anterior), sendo esta incidência semelhante à de McALPINE ${ }^{5}$. BARNER 1, ao comentar o trabalho de Oksner e Mills, afirmou ter-se deparado também com três casos de coronária direita intra-atrial.

PARSONNET ${ }^{10}$, em breve relato, faz observaçōes sobre a maneira de abordagem das artérias coronárias intracavitárias em operaçōes de revascularizaçāo miocárdica. GREEN ${ }^{4}$ nāo concorda com a denominação de artéria coronária intracavitária, afirmando que o posicionamento da artéria é intramural, variando apenas a profundidade da
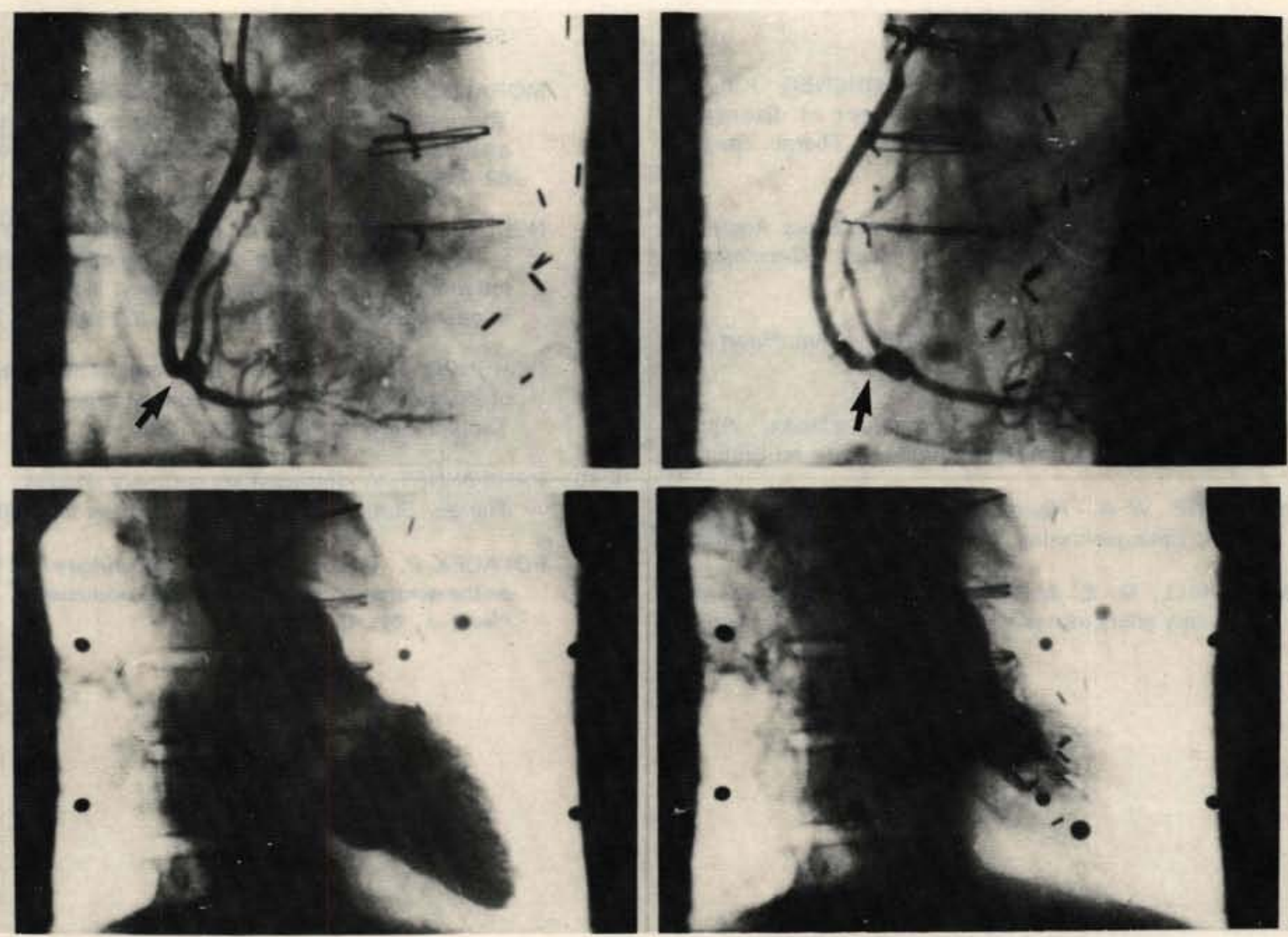

Fig. 4 - F.A.S. - 58a - Masc. - Mulato - RG. 5.039.896-H - Cinecoronariografia pós-operatória, mostrando a ponte de veia safena para artéria coronária direita em $O A E$ e $O A D$, e a ventriculografia esquerda. Nota-se discreta constricção da veia safena no local de emergência do átrio direito (seta). 
LOURENÇĀO JÚNIOR, A.; DALLAN, L. A.; OLIVEIRA, S. A.; JATENE, A. D. - Revascularizaçāo da artéria coronária direita intra-atrial. Rev. Bras. Cir. Cardiovasc. 5(3): 179-182, 1990.

localizaçāo da artéria no miocárdio.

Um fator importante é que a localização intracavitária das artérias coronárias somente é diagnosticada no intra-operatório; a cinecoronariografia pós-operatória (Figura 4) não trouxe subsídios para se definir o trajeto intracavitário e, mesmo, a avaliação retrospectiva da cinecoronariografia pré-operatória (Figura 1) não pode mostrar qualquer indício de tal localização.

Apesar de ter uma incidência bastante baixa (em torno de $0,1 \%$ ), o cirurgião deve estar atento para diagnosticar esta variação anatômica.

\section{RBCCV 44205-122}

LOURENÇĀO JÚNIOR, A.; DALLAN, L. A.; OLIVEIRA, S. A.; JATENE, A. D. - Revascularization of intracavitary right coronary artery. Rev. Bras. Cir. Cardiovasc., 5(3): 179-182, 1990.

ABSTRACT: The right coronary artery, during your course in the right atrioventricular sulcus, can sometimes penetrate the right atrial cavity. This anatomical variety can modify the surgical tactics in aortocoronary by-pass surgery. In this paper we present a patient in whom the by-pass with saphenous vein graft to the right coronary artery was made in right intracavitary position.

DESCRIPTORS: coronary artery, revascularization; myocardial revascularization, surgery.

\section{REFERÊNCIAS BIBLIOGRÁFICAS}

1 BARNER, H. B. - Comentário em OCHSNER, J. L. \& MILLS, N. L. Surgical manegement of diseased intravitary coronary arteries. Ann. Thorac. Surg., 38: $361-370,1984$.

2 CHEITLIN, M. D. - The intramural coronary artery. Another cause for sudden death with exercice? Circulation, 62: 238-242, 1980.

3 GEIRINGER, E . - The mural coronary. Am. Heart J., 41: 359-368, 1951.

4 GREEN, G. E. - Intracavitary coronary arteries. Ann. Thorac. Surg., 40: 99-109, 1985. (Carta ao Editor).

5 MCALPINE, W. A. - Heart and coronary arteries. New York, Springer-Verlag, 1975. Cap. 13, p. 179-191.

6 MARSHALL, M. E. \& HEADLEY, R. N. - Intramural coronary artery as a cause of unstable angina pectoris.
South Med. J., 71: 1304-1309, 1978.

7 MORALES, A. Z.; ROMANELLI R.; BOUCEK, R. J. The mural left anterior descendig coronary artery, atenuous exercise and sudden death. Circulation, 62: 230-239, 1980.

8 NOBLE, J.; BOURASSA, M. G.; PETTICLERC, R.; DYRDA, I. - Myocardial bridingig and milking effect of the left anterior descending coronary artery. Normal variant or obstruction? Am. J. Cardiol., 37: 993-999, 1976.

9 OCHSNER, J. L. \& MILLS, N. L. - Surgical management of diseased intracavitary coronary arteries. Ann. Thorac. Surg., 38: 356-362, 1984.

10 PARSONNET, V. - Intracavitary coronary arteries Ann. Thorac. Surg., 40: 206, 1985. (Cartas ao Editor).

11 POLACEK, P. - Relation of myocardial bridges and loops on the coronary arteries to coronary occlusions. Am. Heart J., 61: 44-50, 1961. 